\title{
ON THE EMBEDDING PROBLEM OF CENTRAL CYCLIC EXTENSIONS OF ABELIAN GROUPS*
}

\section{IVo M. Michailov, IVAylo A. Dimitrov, IVAn S. IVANov}

ABSTRACT: In this report we find the obstruction of the embedding problem related to a central cyclic extension of an arbitrary abelian group.

KEYWORDS: embedding problem, Galois group, field extension, embedding obstruction

2020 Math. Subject Classification: 12 F12

\section{$1 \quad$ Introduction}

Let $k$ be arbitrary field and let $H$ be a non simple group. Assume that $A$ is a normal subgroup of $H$. Then the realizability of the quotient group $G=H / A$ as a Galois group over $k$ is a necessary condition for the realizability of $H$ over $k$. In this way arises the next generalization of the inverse problem in Galois theory - the embedding problem of fields.

Let $K / k$ be a Galois extension with Galois group $G$, and let

$$
1 \longrightarrow A \longrightarrow H \stackrel{\alpha}{\longrightarrow} G \longrightarrow 1,
$$

be a group extension, i.e., a short exact sequence. Solving the embedding problem related to $K / k$ and (1.1) consists of determining whether or not there exists a Galois algebra (called also a weak solution) or a Galois extension (called a proper solution) $L$, such that $K$ is contained in $L, H$ is isomorphic to $\operatorname{Gal}(L / k)$, and the homomorphism of restriction to $K$ of the automorphisms from $H$ coincides with $\alpha$. We denote the so formulated embedding problem by $(K / k, H, A)$. We call the group $A$ the kernel of the embedding problem.

*This paper is (partially) supported by a project No. RD-08-129/04.02.2021 of Shumen University. 
A well known criterion for solvability is obtained by using the Galois group $\Omega_{k}$ of the algebraic separable closure $\bar{k}$ over $k$.

Theorem 1.1. [1, Theorem 1.15.1] The embedding problem $(K / k, H, A)$ is weakly solvable if and only if there exists a homomorphism $\delta: \Omega_{k} \rightarrow$ $H$, such that $\alpha \cdot \delta=\varphi$, where $\varphi: \Omega_{k} \rightarrow G$ is the natural epimorphism. The embedding problem is properly solvable if and only if among the homomorphisms $\delta$, there exists an epimorphism.

Given that the kernel $A$ of the embedding problem is abelian, another well known criterion holds. We can define an $G$-module structure on $A$ by $a^{\rho}=\bar{\rho}^{-1} a \bar{\rho}(\bar{\rho}$ is a pre-image of $\rho \in G$ in $H)$.

Corollary 1.2. [1, Theorem 13.3.2] Let $A$ be an abelian group and let $c$ be the 2-coclass of the group extension (1.1) in $H^{2}(G, A)$. Then the embedding problem $(K / k, H, A)$ is weakly solvable if and only if $\inf _{G}^{\Omega_{k}}(c)=0$

Next, let $K$ contain a primitive root of unity of order equal to the order of the kernel $A$. Then we can define the character group $\hat{A}=$ $\operatorname{Hom}\left(A, K^{*}\right)$ and make it an $G$-module by ${ }^{\rho} \chi(a)=\chi\left(a^{\rho}\right)^{\rho^{-1}}$, for $\chi \in \hat{A}$, $a \in A, \rho \in G$.

Let $\mathbb{Z}[\hat{A}]$ be the free abelian group with generators $e_{\chi}$ (for $\chi \in \hat{A}$ ). We make it an $G$-module by ${ }^{\rho} e_{\chi}=e^{\rho_{\chi}}$. Then there exists an exact sequence of $G$-modules

$$
0 \longrightarrow V \longrightarrow \mathbb{Z}[\hat{A}] \stackrel{\pi}{\longrightarrow} \hat{A} \longrightarrow 0,
$$

where $\pi$ is defined by $\pi\left(\sum_{i} k_{i} e_{\chi_{i}}\right)=\prod_{i} \chi_{i}^{k_{i}}$ where $k_{i} \in \mathbb{Z}$.

We can clearly consider all $G$-modules as $\Omega_{k}$-modules. The exact sequence (1.2) then implies the exact sequence

$$
0 \longrightarrow A \simeq \operatorname{Hom}\left(\hat{A}, \bar{k}^{\times}\right) \longrightarrow \operatorname{Hom}\left(\mathbb{Z}[\hat{A}], \bar{k}^{\times}\right) \longrightarrow \operatorname{Hom}\left(V, \bar{k}^{\times}\right) \longrightarrow 0 .
$$


Since $H^{1}\left(\Omega_{k}, \operatorname{Hom}\left(\mathbb{Z}[\hat{A}], \bar{k}^{\times}\right)\right)=0($ see $[1, \S 3.13 .3])$, we obtain the following exact sequence

$0 \longrightarrow H^{1}\left(\Omega_{k}, \operatorname{Hom}\left(V, \bar{k}^{\times}\right)\right) \stackrel{\beta}{\longrightarrow} H^{2}\left(\Omega_{k}, A\right) \stackrel{\gamma}{\longrightarrow} H^{2}\left(\Omega_{k}, \operatorname{Hom}\left(\mathbb{Z}[\hat{A}], \bar{k}^{\times}\right)\right)$.

We call the element $\eta=\gamma \bar{c}$ the (first) obstruction. The condition $\eta=0$ clearly is necessary for the solvability of the embedding problem $(K / k, H, A)$. This is the well-known compatibility condition found by Faddeev and Hasse. In general it is not a sufficient condition for solvability. Indeed if we assume that $\eta=0$, then there appears a second obstruction, namely $\xi \in H^{1}\left(\Omega_{k}, \operatorname{Hom}\left(V, \bar{k}^{\times}\right)\right)$such that $\beta(\xi)=\bar{c}$. Thus, in order to obtain a necessary and sufficient condition we must have both $\eta=0$ and $\xi=0$. The second obstruction is very hard to calculate explicitly, though. That is why embedding problems for which $H^{1}\left(\Omega_{k}, \operatorname{Hom}\left(V, \bar{k}^{\times}\right)\right)=0$ are of special interest. This condition turns out to be fulfilled in a number of cases, e.g. for the Brauer problems discussed in the next section.

\section{Embedding obstructions for Brauer problems}

Let us begin with the so called Brauer problem. The embedding problem $(K / k, H, A)$ is called Brauer if $\hat{A}$ is a trivial $G$-module. Then we have the well known.

Theorem 2.1. ([1, Theorem 3.1] The compatibility condition for the Brauer problem $(K / k, H, A)$ is necessary and sufficient for its weak solvability.

Let $q \geq 2$ be a natural number, let $k$ be arbitrary field of characteristic relatively prime to $q$, containing a primitive $q$ th root of unity $\zeta$, and put $\mu_{q}=\langle\zeta\rangle$. Let $K$ be a Galois extension of $k$ with Galois group $G$. Consider the group extension

$$
1 \longrightarrow\langle\varepsilon\rangle \longrightarrow H \longrightarrow G \longrightarrow 1
$$


where $\varepsilon$ is a central element of order $q$ in $H$. We are going to identify the groups $\langle\varepsilon\rangle$ and $\mu_{q}$, since they are isomorphic as $G$-modules.

Assume that $c \in H^{2}\left(G, \mu_{q}\right)$ is the 2-coclass corresponding to the group extension (2.1). The obstruction to the embedding problem $(K / k$, $\left.H, \mu_{q}\right)$ we call the image of $c$ under the inflation map inf $\Omega_{G}^{\Omega_{k}}: H^{2}\left(G, \mu_{q}\right) \rightarrow$ $H^{2}\left(\Omega_{k}, \mu_{q}\right)$.

Note that we have the standard isomorphism of $H^{2}\left(\Omega_{k}, \mu_{q}\right)$ with the $q$-torsion in the Brauer group of $k$ induced by applying $H^{*}\left(\Omega_{k}, \cdot\right)$ to the $q$-th power exact sequence of $\Omega_{k}$-modules $1 \longrightarrow \mu_{q} \longrightarrow \bar{k}^{\times} \longrightarrow$ $\bar{k}^{\times} \longrightarrow 1$. In this way, the obstruction equals the equivalence class of the crossed product algebra $(G, K / k, \bar{c})$ for any $\bar{c} \in c$. Hence we may identify the obstruction with a Brauer class in $\operatorname{Br}_{q}(k)$.

Note that we have an injection $\mu_{q} \hookrightarrow K^{\times}$, which induces a homomorphism $v: H^{2}\left(G, \mu_{q}\right) \rightarrow H^{2}\left(G, K^{\times}\right)$. Then the obstruction is equal to $v(c)$, since there is an isomorphism between the relative Brauer group $\operatorname{Br}(K / k)$ and the group $H^{2}\left(G, K^{\times}\right)$.

Clearly, the problem $\left(K / k, H, \mu_{q}\right)$ is Brauer, so from the proof of Theorem 2.1 given in the paper [6] it follows that $H^{1}\left(\Omega_{k}, \operatorname{Hom}\left(V, \bar{k}^{\times}\right)\right)=$ 0 . Hence the homomorphism $\gamma: H^{2}\left(\Omega_{k}, A\right) \rightarrow H^{2}\left(\Omega_{k}, \operatorname{Hom}\left(\mathbb{Z}[\hat{A}], \bar{k}^{\times}\right)\right)$ is an injection. Therefore, the problem is solvable if and only if the (first) obstruction is split.

More generally, the following result holds.

Theorem 2.2. Let $c$ be the 2-coclass in $H^{2}\left(G, \mu_{q}\right)$, corresponding to the group extension (2.1). Then the embedding problem $\left(K / k, H, \mu_{q}\right)$ is weakly solvable if and only if $v(c)=1$. If $\mu_{q}$ is contained in the Frattini subgroup $\Phi(H)$ of $H$, then the condition $v(c)=1$ is sufficient also for the proper solvability of the problem $\left(K / k, H, \mu_{q}\right)$ (see [1, 1.6 , Cor. 5]).

Remark. The related terms weak solvability and Galois algebras were introduced in order to avoid the trouble of describing some very rare exceptions. For example the embedding problem related to the split 
exact sequence $1 \rightarrow C_{2} \rightarrow C_{2} \times C_{2} \rightarrow C_{2} \rightarrow 1$ is 'almost' always solvable in term of fields. We need just to suppose that $\left|k^{*} / k^{* 2}\right| \geq 4$ so that there exist $a, b \in k$ such that $k(\sqrt{a}, \sqrt{b})$ is a $C_{2} \times C_{2}$ extension. However, formally speaking, it is possible that $\left|k^{*} / k^{* 2}\right|<4$ and then obviously we can not define a $C_{2} \times C_{2}$ extension. We can instead define a Galois algebra with Galois group $C_{2} \times C_{2}$ and say that the problem is always weakly solvable.

The main goal is to decompose the obstruction to any $\mu_{q}$ - embedding problem as a product of classes of cyclic algebras. We denote by $(a, b ; \zeta)_{q, k}$ (or just $\left.(a, b)_{q}\right)$ the equivalence class of the cyclic algebra which is generated by $i_{1}$ and $i_{2}$, such that $i_{1}^{q}=b, i_{2}^{q}=a$ and $i_{1} i_{2}=\zeta i_{2} i_{1}$. For $q=2$ we have the quaternion class $(a, b ;-1)$, commonly denoted by $(a, b)$. The first author proved some partial results for $p$-groups in $[2,3,4,5]$ but now we are able to find the obstructions for any group of nilpotency class $\leq 2$.

Let $q \geq 2$ and $n_{1} \leq n_{2} \leq \cdots \leq n_{t}$ be natural numbers. Let $L / K$ be a $G \simeq \prod_{i=1}^{t} C_{n_{i}}$ extension. Assume that for all $i, K$ contains a primitive $n_{i^{-}}$ th root of unity $\zeta_{n_{i}}$ and a primitive $q$-th root of unity $\zeta$. Let $K_{i}=K\left(\sqrt[n_{i}]{a_{i}}\right)$ be the subextension corresponding to the factor $C_{n_{i}}$ for $i=1, \ldots, t$ and some $a_{i} \in K^{\times}$. (That is, $K_{i}$ is the fixed subfield of $\prod_{j \neq i} C_{n_{j}}$.) Let $\sigma_{i}$ be the generator of $C_{n_{i}}$ for $i=1, \ldots, t$. We have that $\sigma_{j} \sqrt[n_{i}]{a_{i}}=\zeta_{n_{i}}^{\delta_{i j}} \sqrt[n_{i}]{a_{i}}(\delta$ is the Kronecker delta).

Theorem 2.3. Let $L / K$ be $a G \simeq \prod_{i=1}^{t} C_{n_{i}}$ extension as described above. Let

$$
1 \longrightarrow \mu_{q} \simeq\langle\zeta\rangle \longrightarrow H \longrightarrow G \simeq \prod_{i=1}^{t} C_{n_{i}} \longrightarrow 1
$$

be a central group extension with cohomology class $\gamma \in H^{2}\left(G, \mu_{q}\right)$. Let $s_{1}, \ldots, s_{t}$ be the pre-images of $\sigma_{1}, \ldots, \sigma_{t}$, let $d_{i j} \in\{0, \ldots, q-1\}$ be given by $s_{i} s_{j}=\zeta^{d_{j i}} s_{j} s_{i}$, and let $s_{i}^{n_{i}}=\zeta^{m_{i}}$ for $i=1, \ldots, t ; m_{i} \in\{0, \ldots, q-1\}$. 
Then $q$ divides $d_{i j} n_{i}$ for all $i, j: j \neq i$, and the obstruction to the weak solvability of the embedding problem $(L / K, H, \mu)$ given by $\gamma$ is

$$
\prod_{i=1}^{t}\left(a_{i}, \zeta^{m_{i}}\right)_{n_{i}} \cdot \prod_{i<j}\left(a_{j}, a_{i}\right)_{n_{j}}^{d_{i j} n_{i} / q} .
$$

If $\zeta \in\left\langle s_{1}, \ldots, s_{t}\right\rangle$ then the obstruction is for the proper solvability.

Proof. First, we are going to show that the existence of the group extension (2.2) implies that $q$ must divide $d_{j i} n_{i}$ for all $i, j: j \neq i$. Since $H$ is nilpotent of class $\leq 2$, we have the commutation rule $[x y, z]=[x, z][y, z]$ for all $x, y, z \in H$. In particular, we have $\zeta^{d_{i i} n_{i}}=\left[s_{i}, s_{j}\right]^{n_{i}}=\left[s_{i}^{n_{i}}, s_{j}\right]=$ $\left[\zeta^{m_{i}}, s_{j}\right]=1$, so $q$ must divide $d_{j i} n_{i}$ for all $i, j: j \neq i$. We can write $d_{j i} n_{i}=q z_{j i}$ for some integer $z_{j i}$ and for all $i, j: j \neq i$. Denote by $\zeta_{d_{j i} n_{i}}$ a primitive $d_{j i} n_{i}$-th root of unity. Then $\zeta_{d_{j i} n_{i}}^{z_{j i}}$ is a primitive $q$-th root of unity and we may assume that $\zeta_{d_{j i} n_{i}}^{z_{j i}}=\zeta$. Similarly, we may assume that $\zeta_{n_{i}}=\zeta_{d_{j i} n_{i}}^{d_{j i}}$. Hence $\zeta_{n_{i}}^{z_{j i}}=\zeta_{d_{j i} n_{i}}^{d_{j i} z_{j i}}=\zeta^{d_{j i}}$ for all $i \neq j$.

We can assume that $d_{i j}=-d_{j i}$ for all $i \neq j$, since $\zeta^{d_{i i}}=\left[s_{i}, s_{j}\right]$ and $\zeta^{d_{i j}}=\left[s_{j}, s_{i}\right]=\left[s_{i}, s_{j}\right]^{-1}=\zeta^{-d_{j i}}$. From the above considerations, we have that $n_{j} z_{j i}=n_{j} d_{j i} n_{i} / q=-\left(n_{j} d_{i j} / q\right) n_{i}$, so $n_{i}$ divides $n_{j} z_{j i}$ for all $j \neq i$.

Let $\mathscr{A}=(L, G, \zeta)$ be the crossed product algebra related to the embedding problem $(L / K, H, \mu)$. Denote $G_{1}=\left\langle\sigma_{1}, \ldots, \sigma_{t-1}\right\rangle$ and $L_{1} / K$ $=K\left(\sqrt[n_{1}]{a_{1}}, \ldots, n_{t} \sqrt[1]{a_{t-1}}\right) / K$. The crossed product algebra $\mathscr{B}=\left(L_{1}, G_{1}\right.$, $\zeta)$ is included in $\mathscr{A}$, therefore $\mathscr{A}$ is a tensor product of $\mathscr{B}$ and the centralizer of $\mathscr{B}$ in $\mathscr{A}: \mathscr{A}=\mathscr{B} \otimes_{K} C_{\mathscr{A}}(\mathscr{B})$.

Now, consider the subalgebra

$$
\mathscr{C}=K\left[\sqrt[n_{t}]{a_{t}}, \prod_{i=1}^{t-1} \sqrt[n_{i}]{a_{i}}-z_{t i} \cdot s_{t}\right]
$$

in $\mathscr{A}$. We have that $\left(\prod_{i=1}^{t-1} \sqrt[n_{i}]{a_{i}}-z_{t i} \cdot s_{t}\right)^{n_{t}}=\zeta^{m_{t}} \prod_{i=1}^{t-1} a_{i}^{-z_{t i} n_{t} / n_{i}} \in K,\left(\sqrt[n_{t}]{a_{t}}\right)^{n_{t}}$ $=a_{t}$ and $\prod_{i=1}^{t-1} \sqrt[n_{i}]{a_{i}}-z_{t i} \cdot s_{t} \cdot \sqrt[n_{t}]{a_{t}}=\zeta_{n_{t}} \sqrt[n_{t}]{a_{t}} \cdot \prod_{i=1}^{t-1} \sqrt[n_{i}]{a_{i}}-z_{t i} \cdot s_{t}$. Therefore, 
$\mathscr{C}$ is the cyclic algebra

$$
\mathscr{C} \simeq\left(a_{t}, \zeta^{m_{t}} \prod_{i=1}^{t-1} a_{i}^{-z_{i t} n_{t} / n_{i}}\right)_{n_{t}}
$$

Next, we will show that $\mathscr{C}$ is in fact the centralizer $C_{\mathscr{A}}(\mathscr{B})$. Indeed, for $1 \leq \kappa \leq t-1$ we have

$$
\begin{aligned}
& s_{\kappa}\left(\prod_{i=1}^{t-1} \sqrt[n_{i}]{a_{i}}-z_{t i}\right) s_{t}=\left(\prod_{i=1}^{t-1} \zeta_{n_{i}}^{\delta_{i \kappa}\left(-z_{t i}\right)} \sqrt[n_{i}]{a_{i}}-z_{t i}\right) s_{\kappa} s_{t} \\
& =\left(\prod_{i=1}^{t-1} \zeta_{n_{i}}^{\delta_{i \kappa}\left(-z_{t i}\right)} \sqrt[n_{i}]{a_{i}}-z_{t i}\right) \zeta^{-d_{\kappa t}} s_{t} s_{\kappa} \\
& =\zeta^{-d_{\kappa t}} \zeta^{\sum_{i=1}^{t-1} \delta_{i \kappa}\left(-d_{t i}\right)}\left(\prod_{i=1}^{t-1} \sqrt[n_{i}]{a_{i}^{-}}-z_{t i}\right) s_{t} s_{\kappa}=\left(\prod_{i=1}^{t-1} \sqrt[n_{i}]{a_{i}}-z_{t i}\right) s_{t} s_{\kappa},
\end{aligned}
$$

since $\zeta_{n_{i}}^{-z_{t i}}=\zeta^{-d_{t i}}=\zeta^{d_{i t}}$ and $\sum_{i=1}^{t} \delta_{i \kappa}\left(-d_{t i}\right)=d_{\kappa t}$. Therefore,

$$
[\mathscr{A}]=[\mathscr{B}]\left(a_{t}, \zeta^{m_{t}} \prod_{i=1}^{t-1} a_{i}^{-z_{t i}}\right)_{n_{t}}=[\mathscr{B}]\left(a_{t}, \zeta^{m_{t}}\right)_{n_{t}} \prod_{i=1}^{t-1}\left(a_{t}, a_{i}\right)_{n_{t}}^{d_{i t} n_{i} / q}
$$

and the theorem follows by induction.

It is not hard to see that $\zeta \in\left\langle s_{1}, \ldots, s_{t}\right\rangle$ if and only if $\zeta$ is in the Frattini subgroup $\Phi(H)$. From Theorem 2.2 now it follows that the obstruction is also for the proper solvability.

The above theorem includes the non trivial embedding problem for cyclic groups (here the product $\prod_{i<j}\left(a_{j}, a_{i}\right)_{n_{j}}^{d_{i j} n_{i} / q}$ is 1 , because $d_{i j}=$ 0 for all $i \neq j$ ). As for the case when the extension (2.2) is split, the group $H$ is abelian and is a direct product of the kernel and the quotient group, since the kernel is central. This problem has a trivial (splitting) obstruction and is always weakly solvable. 
Finally, we would like to remark that Theorem 2.3 can be used for the inverse problem for any group $H$ of nilpotency class 2 over any field $k$, containing a primitive root of unity of degree the exponent of $H$. The reason is that any such group $H$ is a pullback of groups that are cyclic extension of an abelian group. We are going to give some more details now.

Let $\varphi^{\prime}: H^{\prime} \rightarrow G$ and $\varphi^{\prime \prime}: H^{\prime \prime} \rightarrow G$ be homomorphisms with kernels $N^{\prime}$ and, respectively, $N^{\prime \prime}$. The pullback of the pair of homomorphisms $\varphi^{\prime}$ and $\varphi^{\prime \prime}$ is the subgroup in $H^{\prime} \times H^{\prime \prime}$ of all pairs $\left(\sigma^{\prime}, \sigma^{\prime \prime}\right)$, such that $\varphi^{\prime}\left(\sigma^{\prime}\right)=\varphi^{\prime \prime}\left(\sigma^{\prime \prime}\right)$. The pullback is denoted by $H^{\prime} \curlywedge H^{\prime \prime}$. It is also called the direct product of the groups $H^{\prime}$ and $H^{\prime \prime}$ with amalgamated quotient group $G$ and denoted by $H^{\prime}{ }^{*}{ }_{G} H^{\prime \prime}$.

Next, let $N_{1}=N^{\prime} \times\{1\}$ and $N_{2}=\{1\} \times N^{\prime \prime}$. Then $N_{1}$ and $N_{2}$ are normal subgroups of $H^{\prime} \curlywedge H^{\prime \prime}$, such that $N_{1} \cap N_{2}=\{1\}$. The converse is also true (see [1], I, §12):

Lemma 2.4. Let $N_{1}$ and $N_{2}$ be two normal subgroups of the group $H$, such that $N_{1} \cap N_{2}=\{1\}$. Then $H$ is isomorphic to the pullback $\left(H / N_{1}\right) \curlywedge$ $\left(\mathrm{H} / \mathrm{N}_{2}\right)$.

The application to embedding problems is given by:

Theorem 2.5. ([1, Theorem 1.12]) Let $K / k$ be a Galois extension with Galois group $G$. In the notations of Lemma 2.4, let $G \simeq H / N_{1} N_{2}$ and $H \simeq\left(H / N_{1}\right) \curlywedge\left(H / N_{2}\right)$. Then the embedding problem $\left(K / k, H, N_{1} N_{2}\right)$ is solvable if and only if the embedding problems $\left(K / k, H / N_{1}, N_{2}\right)$ and $\left(\mathrm{K} / \mathrm{k}, \mathrm{H} / \mathrm{N}_{2}, \mathrm{~N}_{1}\right)$ are solvable.

Let $H$ be a nilpotent group of class 2. Then $H / Z(H)$ is abelian, and the center $Z(H)$ can be decomposed into a direct product of cyclic groups, which, of course, are normal in $H$. Therefore $H$ is a pullback of cyclic extensions of $H / Z(H)$, for which we can compute the obstructions. This will give us all the obstructions for the embedding problems with an abelian kernel. 


\section{REFERENCES:}

[1] Ishanov V. V., Lur'e B. B. and Faddeev D. K., "The embedding problem in Galois theory", Amer. Math. Soc., Providence, 1997.

[2] Michailov I., Four non-abelian groups of order $p^{4}$ as Galois groups, $J$. Algebra 307 (2007), 287-299.

[3] Michailov I. , On Galois cohomology and realizability of 2-groups as Galois groups, Cent. Eur. J. Math., 9 (2) (2011), 403-419.

[4] Michailov I., On Galois cohomology and realizability of 2-groups as Galois groups II, Cent. Eur. J. Math., 9 (6) (2011), 1333-1343.

[5] Michailov I. , Galois realizability of groups of orders $p^{5}$ and $p^{6}$, Cent. Eur. J. Math., 11 (5), 2013, 910-923.

[6] Michailov I. and Ziapkov N., Embedding obstructions for the generalized quaternion group, J. Algebra 226 (2000), 375-389.

\section{Ivo M. Michailov}

Faculty of Mathematics and Informatics, Shumen University, Universitetska str. 115, 9700 Shumen, Bulgaria

e-mail: ivo_michailov@yahoo.com

\section{Ivaylo A. Dimitrov}

Faculty of Mathematics and Informatics, Shumen University, Universitetska str. 115, 9700 Shumen, Bulgaria

e-mail: pmg.iv.dimitrov@abv.bg

\section{Ivan S. Ivanov}

Faculty of Mathematics and Informatics, Shumen University, Universitetska str. 115, 9700 Shumen, Bulgaria e-mail: slaveicoveabv.bg 